resection using ESD knives and post-resection closure with OTSC(Group 1), the other was closure with OTSC and secondary EFTR with snare(Group 2).

Results Of 11 patients, 6 cases were in Group 1 and 5 cases in Group 2. The mean time of EFTR procedure was $76.83 \pm$ 34.97 minutes in Group 1 which is significantly longer than that of Group $2(\mathrm{P}=0.0128)$. The mean time of OSTC closure and length of hospital stay of Group 1 were also longer compared to Group 2, but the difference was not significant. Complete resection (R0) and technical success rate of Group 1 and Group 2 were $83.3 \%$ and $100 \%(\mathrm{P}=0.338)$.VAS scores of Group 1 after operation and 24 hours are significantly higher than those of Group $2(\mathrm{P}=0.047$ and $\mathrm{P}=0.009$, respectively). In Group 1, One patient had delayed perforation which led to fever and pneumoperitoneum, and one patient developed abdominal pain.No complications associated with endoscopic procedure was observed in Group 2.

Conclusions EFTR of pre-resection closure is potentially faster and safer compared with the concept of applying closure after EFTR. Larger prospective controlled studies comparing these two techniques are warranted in the future.

\section{IDDF2019-ABS-0044 UTILITY OF INTESTINAL TISSUE BIOPSY XPERT-MTB/RIF IN EVALUATION OF PATIENTS WITH ILEO-CAECAL ULCERS}

Balaji L Bellam*, Harshal Mandavdhare, Kusum Sharma, Siddharth Shukla, Hariom Soni, Praveen Kumar, Harjeet Singh, Kaushal Prasad, Usha Dutta, Vishal Sharma. Postgraduate Institute of Medical Education and Research, India

\subsection{6/gutjnl-2019-IDDFabstracts.126}

Background Ileo-cecal ulcers could result from a number of conditions including intestinal tuberculosis and Crohn's disease. We report the use of GeneXpert Mtb/Rif in the diagnosis of ITB in patients with ileo-cecal ulcers.

Methods One year retrospective study involving patients with ileo-cecal ulcers who underwent GeneXpert Mtb/Rif from the intestinal biopsies taken from ulcers. The results of the test were compared with the final diagnosis. We divided the patients into two groups: those with a final diagnosis of ITB and those with a final diagnosis other than ITB (OTH).

Results Of the 40 patients with ileo-cecal ulcers included, 23 $(57.5 \%)$ were females and the mean age was $32.92 \pm 12.78$ years. Abdominal pain was present in $33(88.5 \%)$ patients and diarrhea in 12 (30\%). Out of 40 patients, 25 had ITB and 15 patients were in the OTH group. The sensitivity, specificity, negative predictive value, the positive predictive value and accuracy of GeneXpert-Mtb/Rif for diagnosis of ITB was $32 \%$ (CI: $14.95-53.50 \%), \quad 100 \% \quad(78.2-100), \quad 46.88 \% \quad(40.27-$ $53.59 \%), 100 \& 57.50$ (40.89-72.89)\% respectively.

Conclusions A positive GeneXpert-Mtb/Rif is helpful in the diagnosis of ITB but the sensitivity is low.

\section{IDDF2019-ABS-0053 COMPARISON OF OUTCOMES OF COLORECTAL POLYPECTOMY USING TRADITIONAL SNARE AND ROTARY SNARE: A PROSPECTIVE RANDOMIZED CONTROLLED TRIAL}

${ }^{1} \mathrm{Lu} \mathrm{Xu}{ }^{*},{ }^{1} Z$ hixing Zhang, ${ }^{1}$ Jiarong Xie, ${ }^{2}$ Lei Xu, ${ }^{2}$ Weihong Wang. 'Ningbo University, College of Medicine, Ningbo, China; ${ }^{2}$ Department of Gastroenterology, Ningbo First Hospital, Ningbo, China

\subsection{6/gutjnl-2019-IDDFabstracts.127}

Background The aim of the current study was to compare the efficacy and safety of polypectomy by using rotary snare vs. traditional snare during colonoscopy.

Methods A single-center randomized controlled trial, which included consecutive participants who were $\geq 18$ years old and detected with polyp(s) during routine colonoscopy between July and September 2018, was conducted. Participants with colorectal polyps were randomized to receive polypectomy using rotary snares or traditional snares. The primary outcome measure was the comparison of the average time of removing a polyp between those two groups. The secondary outcome measure was to compare the polyp resection time by using SMSA (Size, Morphology, Site, and Access) scores.

Results A total of two hundred participants were included in this study. Of them, 100 participants were randomly assigned to the rotary snare group (214 polyps) and the other 100 participants were randomly assigned to the traditional group (232 polyps). The mean resection time was significantly shorter in the rotary snare group than in the traditional snare group (24.41[SD:18.14]seconds vs. 29.53[SD:25.74]seconds, $P=0.021$ ). In the subgroup analysis, the resection time was also shorter in the rotary snare group than the traditional
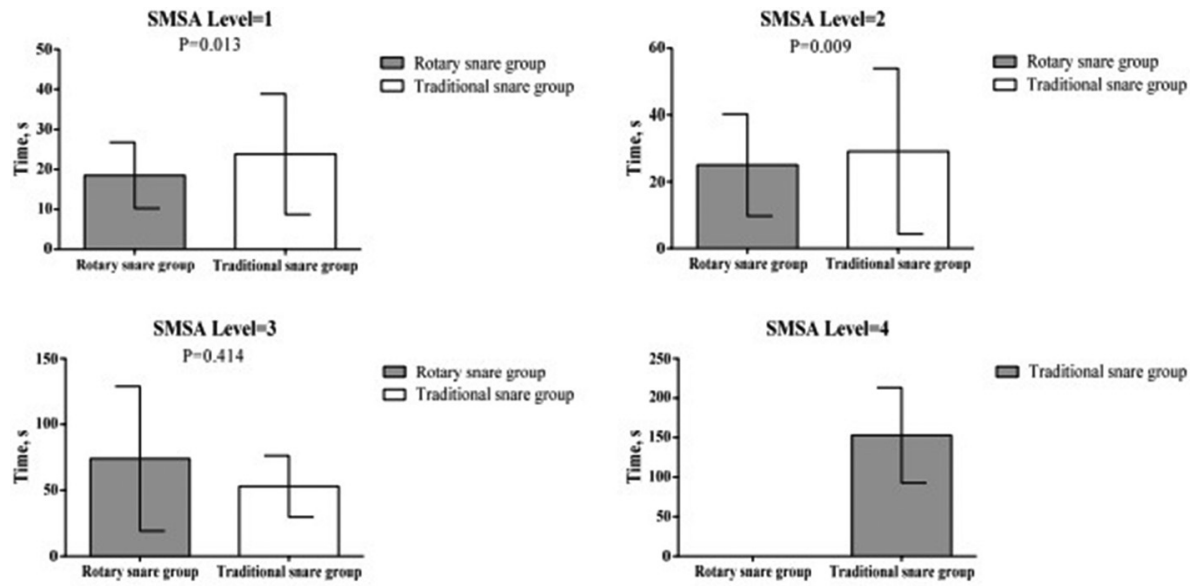

Abstract IDDF2019-ABS-0053 Figure 1 Comparison of polypectomy procedure times in size morphology site and access SMSA score between the traditional snare group and rotary snare group 